\title{
DETERMINING MORPHOMETRIC PROPERTIES OF RADIATA PINE USING LONG WAVE INFRARED SENSING AND BIOLOGICALLY-INSPIRED VISION
}

\author{
Anthony Finn ${ }^{1 *}$, Russell Brinkworth ${ }^{1}$, Daniel Griffiths ${ }^{1}$, Stefan Peters ${ }^{2}$ \\ \{anthony.finn, russell.brinkworth, daniel.griffiths, stefan.peters\}@unisa.edu.au \\ ${ }^{1}$ School of Engineering, University of South Australia, Mawson Lakes, Australia, SA 5095 \\ ${ }^{2}$ School Natural Built Environment, University of South Australia, Mawson Lakes, Australia, SA 5095
}

KEY WORDS: UAVs, remote sensing, image processing, forestry, infrared sensor

\begin{abstract}
:
A miniaturised, high resolution visible and long wave infrared (LWIR) sensor was carried onboard an unmanned aerial vehicle to observe sections of radiata pine forests. The raw irradiance measurements were temporally pre-processed using a biologically-inspired vision (BIV) model to allow information within and across the images to be normalised. This permitted a larger, denser, and more tailored set of key points within the 2D image stacks to be corresponded, thereby improving 3D reconstructions of individual trees derived using structure from motion (SfM). The BIV model comprises multiple layers of processing derived from measured or assumed responses of the photoreceptor cells in the hoverfly. Its pre-processing expands the range of input signal obtained from the LWIR sensor and enhances foreground-background contrast. Morphological image processing techniques were also applied to enhance key image features before structure from motion is applied. The result allows structural properties of individual trees to be characterised in terms of their potential volume and quality; and contrasted with the point clouds obtained from the visible imagery that only depicts the tree canopies.
\end{abstract}

\section{INTRODUCTION}

\subsection{Motivation}

There is a need for forest management and conservation tools that improve workflow efficiency and safety through a combination of timely data acquisition and high spatio-temporal resolution. Such tools should also extend operational reach and access, as well as cut costs by reducing economic losses caused by incorrect decisions based on poor or erroneous data.

In recent decades, conventional forest inventories at various geographical scales have been augmented by using remotely sensed data, such as airborne laser scanning (ALS) and photogrammetry techniques like structure from motion (SfM) (Kwak et al, 2007; Chen et al, 2006; Wallace et al, 2015; Gong et al 2002). High resolution point clouds may also be obtained using sensors carried onboard unmanned aerial vehicles (UAVs); and the data obtained from such approaches examined to morphologically classify the properties and quality of individual trees using shape, structure, and intensity features. Such approaches are frequently augmented with multi- or hyperspectral sensing observations.

The morphometric information obtained has the potential to serve many forest applications (Strigul, 2008; 2012), especially the retrieval of tree quality for planning silvicultural activities and cutting/thinning regimes. Furthermore, from an ecological standpoint, such techniques offer valuable capabilities for monitoring forest regeneration, quantitative analysis of forest structure and dynamics, and evaluation of forest damage.

While still in their operational infancy, these non-invasive UAVbased techniques offer the potential for biophysical attributes such as tree density, and tree characteristics such as height, basal area, stem volume, and crown area to be derived or modelled with high accuracy. Forests, however, due to their heterogeneity and density, are often difficult to observe and assess from the air: they are usually vast and not easily accessible, with tree species varying in shape and size. Moreover, the image capture systems have to deal with very challenging outdoor environments where lighting and contrast can vary over six orders of magnitude, and glare and strong shadow is detrimental to their performance.

\subsection{Long wave infrared sensing of radiata pine}

Although energy exchange takes place throughout an entire volume of forest, the main components occur via a plants' leaves, or in the case of radiata pine, via their needles. The stomata, which are microscopic cells beneath the surface of the needles, exchange water vapour, carbon dioxide, and oxygen with the atmosphere. This has a cooling effect as substantial energy is required to convert liquid water to water vapour. Moreover, as radiata pine do not transpire in the same way, they are typically warmer than the needles.

It is well-known that the intensity of long wave infrared (LWIR) radiation emitted by objects is mainly a function of their temperature. As a result, it is used extensively for multiple purposes in medical, engineering, building and remote sensing applications; and it is well-suited for observing plants whose components exhibit differing thermal structures and such techniques have been extensively reviewed by (Jones, 2004).

The total radiation received by an LWIR sensor, $I_{\text {tot }}$, comes from three sources: the emission of the target object, $I_{t g t}$, the emission reflected by the environment and the target object, $I_{\text {ref }}$, and the emission of the atmosphere, $I_{\text {atm }}$ such that $I_{\text {tot }}=I_{\text {tgt }}+I_{\text {ref }}+$ $I_{\text {atm }}$. Typically, in order to compute each of these components

* Corresponding author (anthony.finn@ unisa.edu.au) 
accurately, a priori knowledge or careful measurement of a target's emissivity is required. Atmospheric temperatures and the amount of energy reflected by the environment must also be determined (Usamentiaga et al., 2014). Undertaking such activities in forest environments is complex and fraught with difficulty.

However, as we are not concerned with measuring foliage or stem temperatures per se, we do not need to determine $I_{t g t}, I_{r e f}$, or $I_{\text {atm }}$ accurately. We can forego calibration procedures and use the sensor solely as a means to detect structures of interest, such as needles, which transpire, or trunks, which do not. We achieve this by simply 'tuning' the image processing appropriately. Thus, while any observed absolute or relative temperature differences of scenes may be biased, we do not concern ourselves with this as we are only interested in local (i.e. regional) image contrast.

Nevertheless, along with the sensitivity, spatial, and thermal resolution of the LWIR sensor, the density and height of trees in the forest, density of foliage on individual trees, tree-camera geometry, and the prevailing meteorological conditions all play a part in determining the extent to which the tree trunks form a contrast against their background; and the precise nature and extent to which each of these has an impact on the result of what follows forms a component of an ongoing research program.

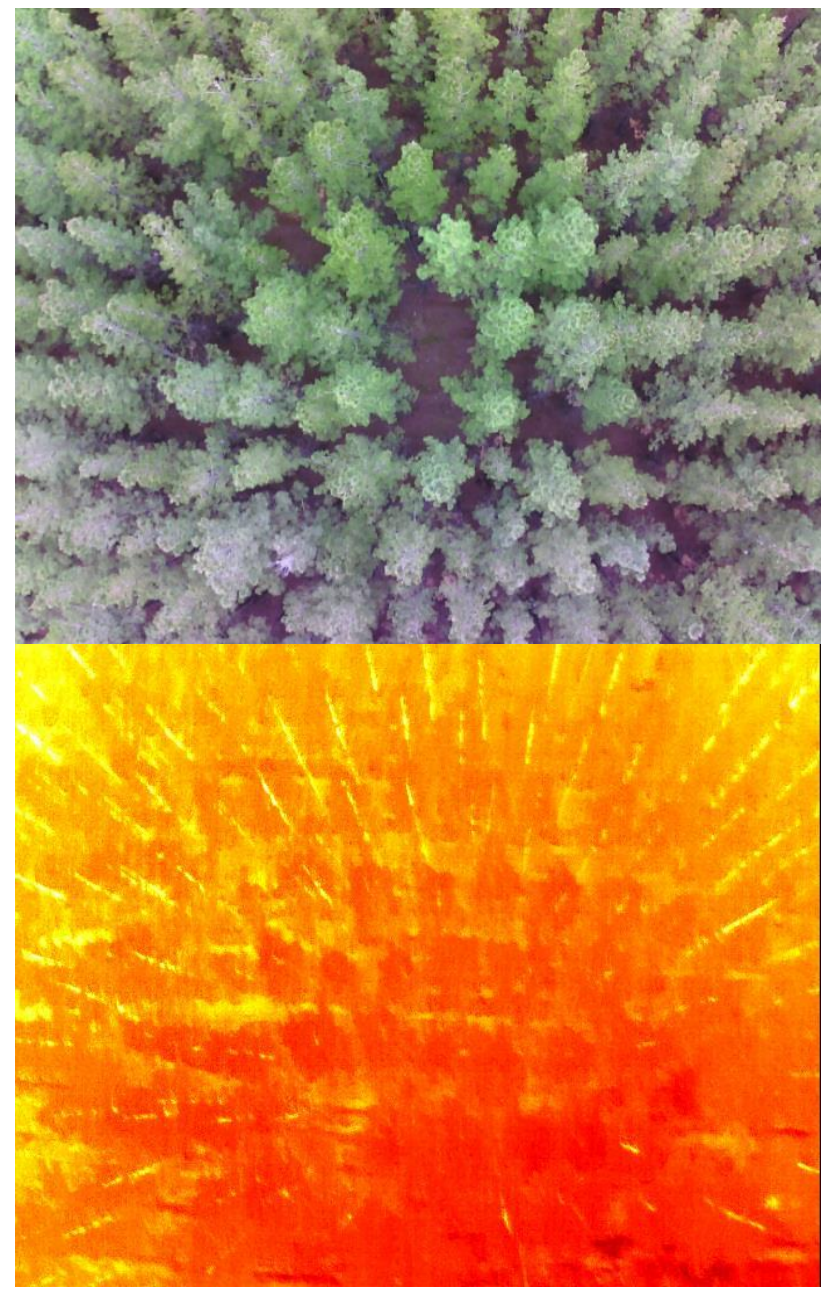

Figure 1: Radiata pine observed in the visible (left) and LWIR (right) spectral bands

An example of the comparable contrasts achievable is shown in Figure 1 . This shows the same scene observed simultaneously by a 5MP oCam visible range camera (left) and an Infratherm P9640 LWIR camera (right). Both sensors were carried onboard a Matrice $600 \mathrm{UAV}$ at a height of about $30 \mathrm{~m}$ above the tops of the trees, which at about $20 \mathrm{~m}$ high. Tree separation is roughly $3 \mathrm{~m}$.
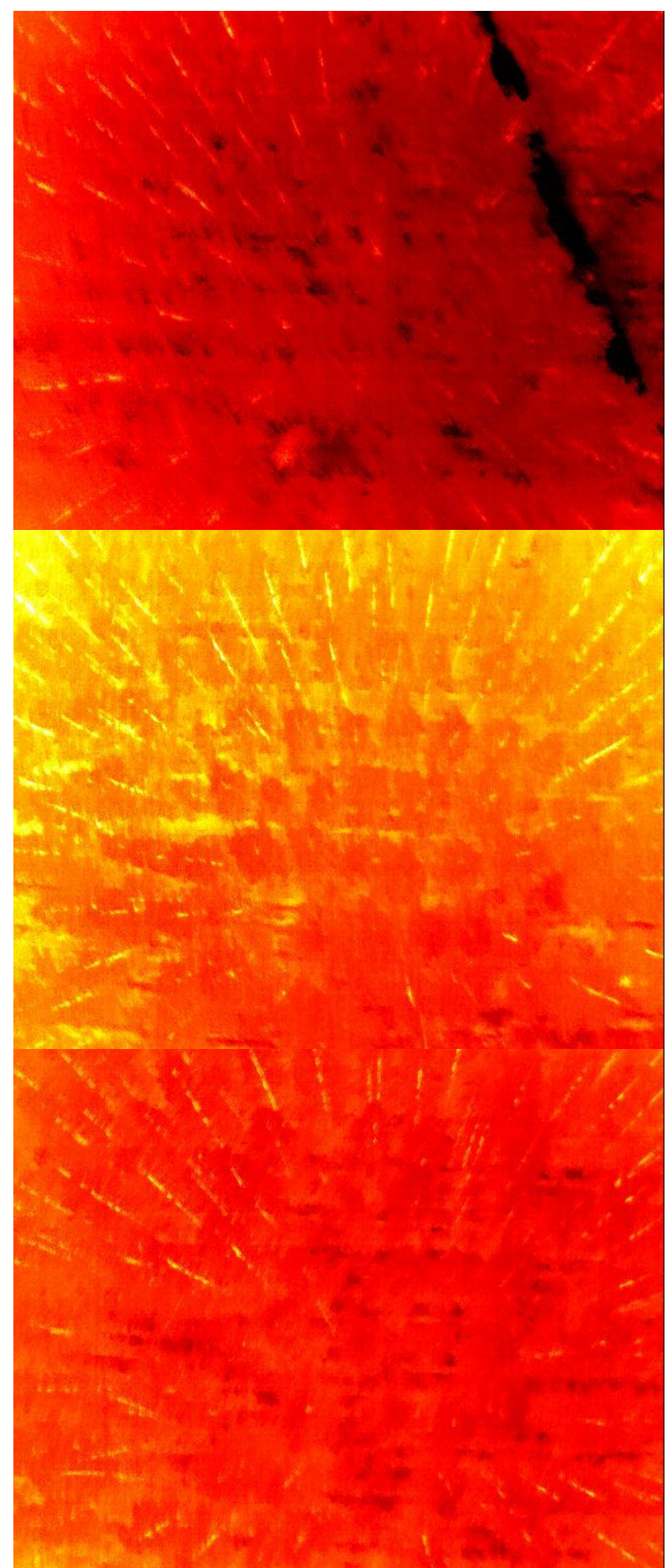

Figure 2: Contrast variations within LWIR observations of radiata pine (the images were taken within 30 s of each other)

As the UAV overflies the forest circumstances and conditions continually alter. So, even if $I_{t g t}$ (effectively trunk temperature) does not change substantially, the reflected irradiance, $I_{r e f}$, and hence total irradiance, $I_{t o t}$, does. This results in significant variation in the perceived or brightness temperature, $T_{B}=$ $\sqrt[4]{\sigma I_{t o t}}$, where $\sigma$ is Stefan's constant, both within and across 
images for the foreground (trunks and stems) as well as background (forest floor, canopy, etc.) - see Figure 2.

As the overarching goal is to combine $2 \mathrm{D}$ imagery observed by the UAVs in a manner that permits the the 3-D structure of the scene to be estimated so that tree properties and quality may be assessed, the information within and across the images must be normalised so that potential key points can be accurately corresponded. To achieve this, and because we found that traditional morphological image processing techniques were inferior, we use a biologically-inspired vision (BIV) model (Brinkworth and O'Carroll, 2009).

\section{IMAGE PROCESSING APPROACH}

The BIV is a multi-stage signal processing pipeline derived from the hover-fly's visual pathways. The class-leading capability of the model has been demonstrated in the fields of motion detection (Brinkworth et al., 2010), object identification (Dowley et al., 2013), optical character recognition (Poursoltan, 2015), and dynamic range compression (Griffiths, 2018).

In its real time implementation, the BIV model has been divided into processing stages based on the source neurology, grouped into the functional pipelines: pre-processing, motion estimation, and target isolation. The first and second stages of the preprocessing pipeline are inspired by the photoreceptor cell (PRC) and lamina monopolar cell (LMC) found within biology. Due to its biologically-inspired nature the BIV model is incredibly tolerant to low-SNR environments (less than 0dB), where competing methods regularly fail.

The PRC component simulates a per-pixel shutter, that performs spatially-invariant, pixel-wise intensity normalisation. This intelligently compresses scene dynamic range. Illumination effects - shadowing and highlights - are then removed via digital integration with adaptive low-pass filtering, enabling consistent representation of objects across multiple lighting scenarios. This multi-level adaptation results in an image with near equivalent signal quality across all pixels, irrespective of pixel intensity. However, this does remove any absolute reference to scene luminance, instead opting preserve and enhance local contrasts.

The second stage of the pre-processing pipeline, the LMC, applies adaptive spatial-temporal filtering to reduce image redundancy. Spatially, this supresses regions with minor contrast via high pass and difference of Gaussian filters, while enhancing edges and features. Temporally, static pixels are supressed over time while image bandwidth is dedicated to dynamic pixels. The LMC high pass filters are leaky in design, and can thus be tuned to enhance or preserve spatial-temporal edges exclusively.

\subsection{Structure from motion}

Structure from motion (SfM) is then applied to the resulting image stack. SfM is extensively described elsewhere (LonguetHiggins, 1981, Furukawa and Ponce, 2010, Hartley, 1997) but essentially comprises two components: camera motion estimation and point cloud reconstruction.

Initially, a sparse set of points are matched across the 2D image stack to find correspondences. Such features are typically extracted using algorithms such as SIFT (scale invariant feature transformation) (Lowe, 1999) and SURF (speeded up robust features) (Bay et al., 2008). The sequence of views are then iteratively processed to track a denser set of points across the views so that the pose of the camera can be established for each image set and-after the relevant coordinate transforms have been accommodated - a dense 3D reconstruction of the scene made. The process of estimating camera motion (and hence point cloud) is generally improved if camera pose is recorded during image capture and applied during SfM computations.
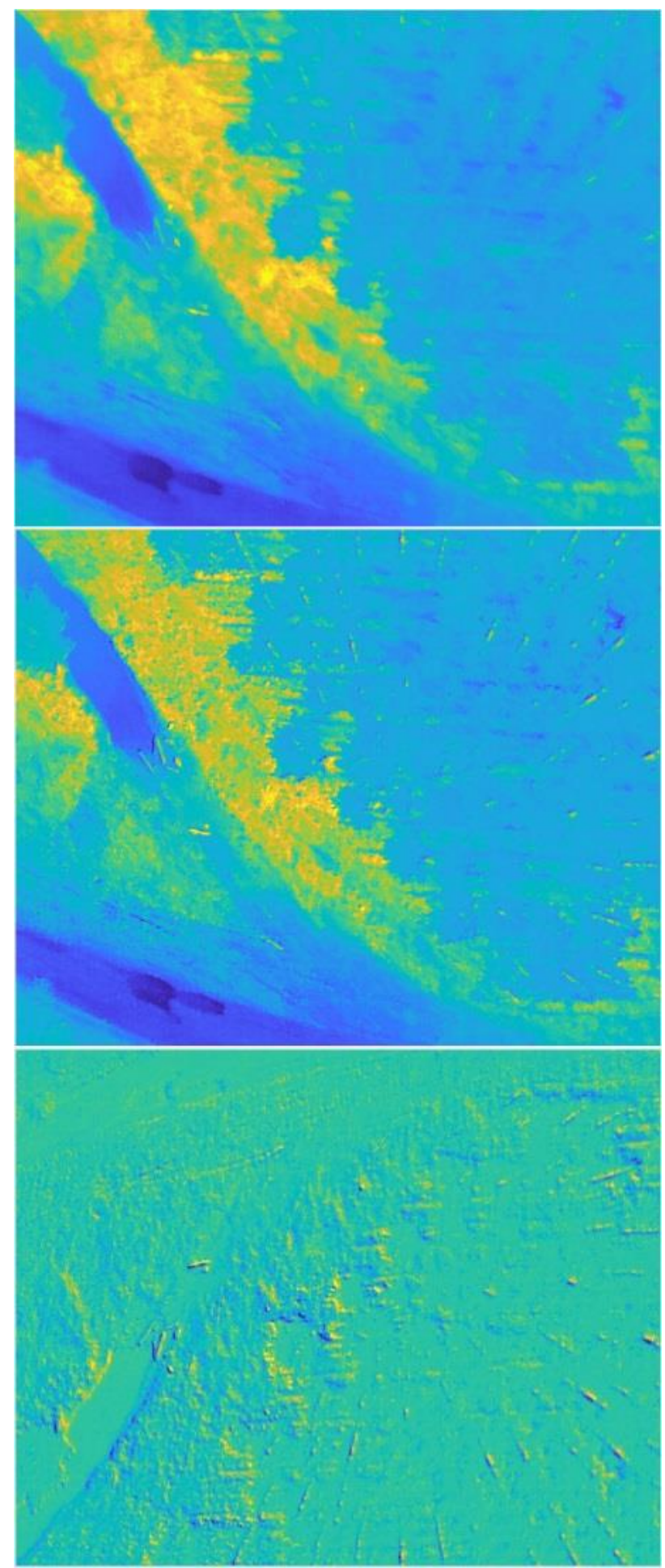

Figure 3: Example of image normalisation and edge enhancement using the BIV model

Over the period of this work we have used three sets of software for obtaining SfM point clouds: a proprietary approach written in MATLAB, Agisoft Photoscan, and Reality Capture. We have not formed a view as to whether any one product provides superior 
performance, except that the commercial packages execute much more efficiently than the bespoke ('home grown') software.

\section{PRELIMINARY RESULTS}

The result of this non-linear, adaptive normalisation process is a 3D, SfM-generated point cloud of tree trunks (Figure 4), which may be combined with point clouds of the canopy obtained from imagery taken of the same trees in the visible range (Figure 5). The LWIR combined with BIV processing thus offers access to tree properties such as volume, height, diameter, crown area, and stem density. The accuracy with which such attributes may be determined forms a part of an ongoing research program.

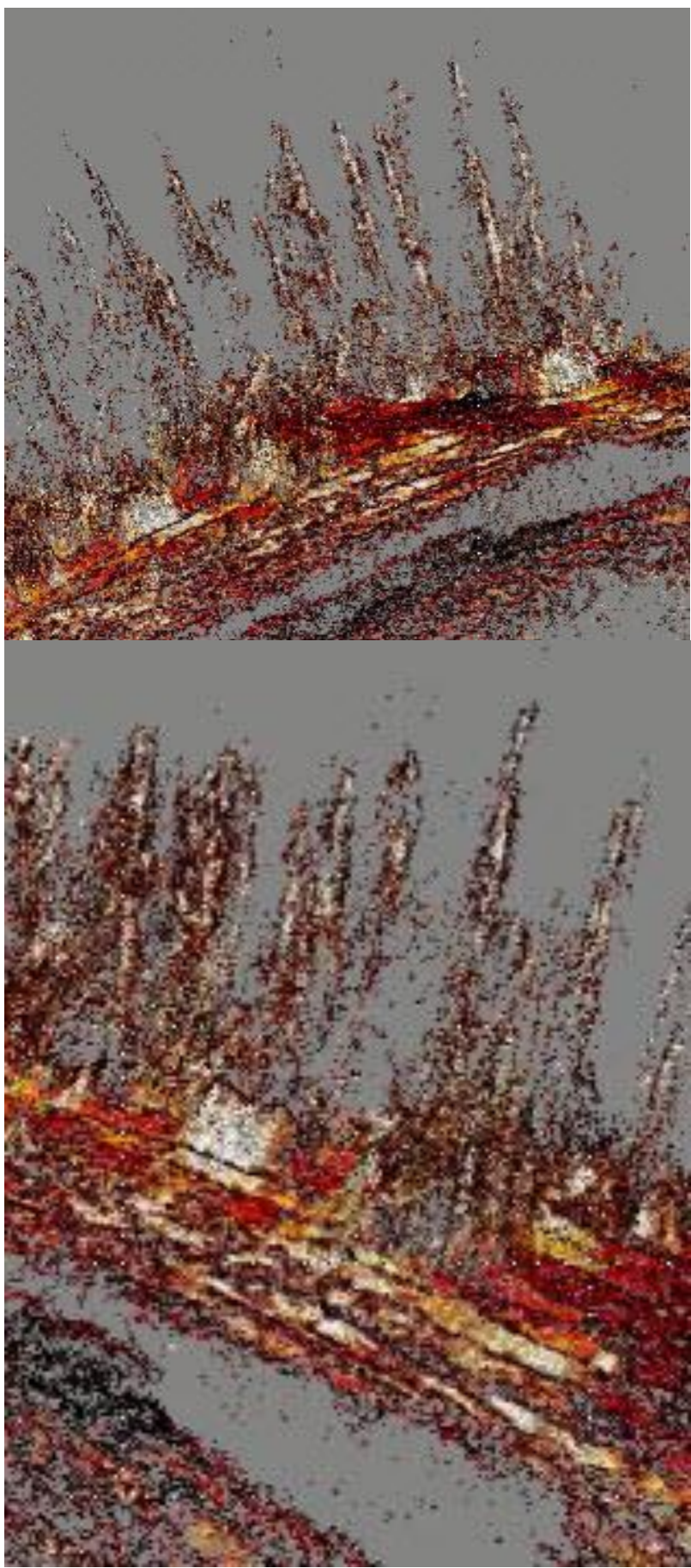

Figure 4: 3D reconstruction of trees observed using LWIR and BIV pre-conditioning with SfM obtained using Agisoft software
A quantitative analysis of the data is ongoing. A large number of trees were observed as part of this study and the LWIR and BIV processing appears able to identify the stems reliably. However, this does not always translate into good SfM data; and omission and commission errors resulting from the processing are yet to be quantified. Nevertheless, we found that without any BIV or other morphological pre-processing generation of the SfM point clouds representing the stem structures of the trees was vastly inferior.

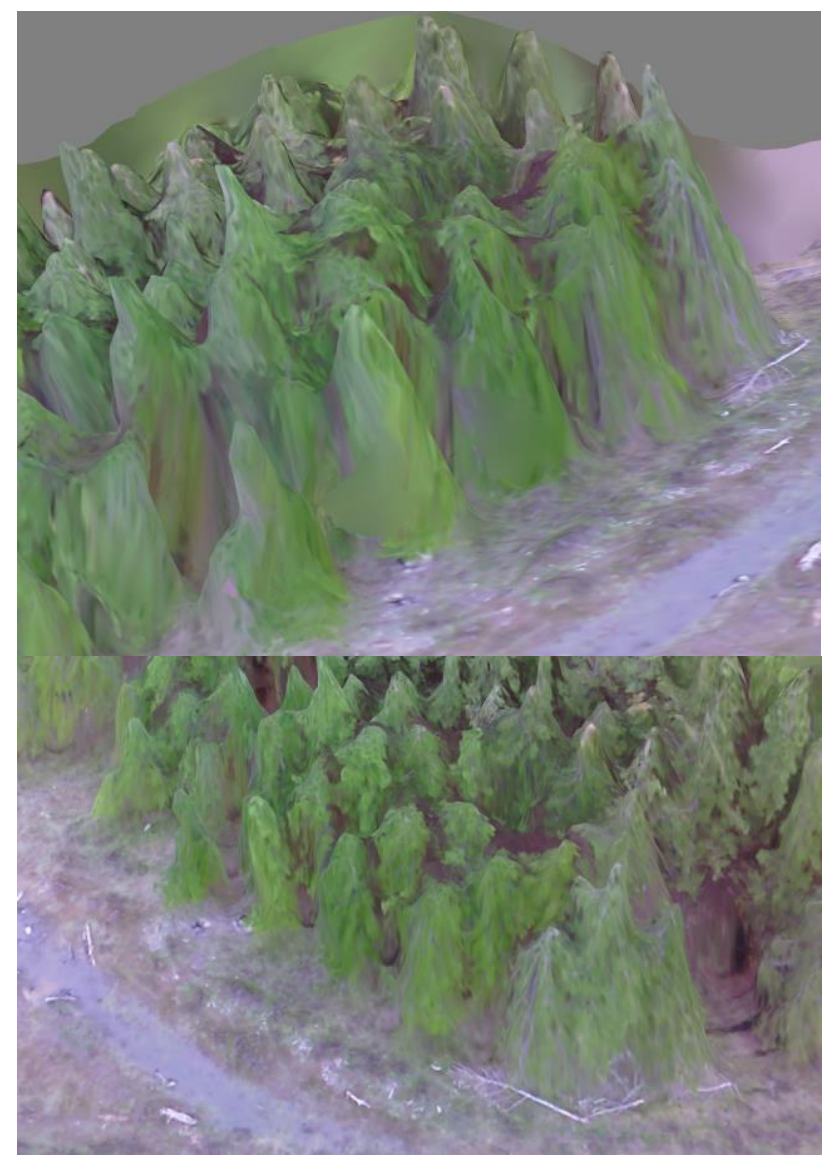

Figure 5: 3D reconstruction of trees observed using VIS with SfM obtained using software by Agisoft

We have yet to determine the impact of tuning specific BIV model parameters on tree detection, and their relationship on SfM reconstruction. From our results, however, it can be concluded that the UAV-based LWIR measurements can be combined with the BIV technique and individual and stand morphometric parameters obtained for the density and type of pine forests observed during these experiments. The possibility of applying the BIV to the image stacks observed on the visible component of the spectrum is also being considered.

\section{SUMMARY}

Irradiance measurements in the $800-1200 \mathrm{~nm}$ band were preconditioned using a BIV model to allow information within and across the observed images to be normalised prior to application of SfM. This enables a more relevant set of key points within the 2D image stacks to be corresponded, thereby improving the 3D reconstructions of individual tree properties.

The BIV model offers greatest benefit in complex, low lit situations or those subject to rapid or unpredictable lighting changes. This typically occurs in the inner regions of densely 
populated forests. The results allow structural properties of individual trees to be characterised in terms of their potential volume and quality; and aligned to and contrasted with the point clouds obtained from visible imagery gathered at the same time and that depicts the tree canopy. This has the potential to augment future research directed towards estimation of above ground biomass and stem volume.

\section{ACKNOWLEDGEMENTS}

This work is supported by the Australian National Institute for Forest Products Innovation (NIFPI). We are grateful to our colleagues Jim O'Hehir (UniSA) and Steve Andriolo (EyeSky) for their extensive technical and trials support.

\section{REFERENCES}

Bay, H., Ess, A., Tuytelaars, T. \& Van Gool, L. (2008) SURF: Speeded Up Robust Features. Computer Vision and Image Understanding 110(3):346-359.

Brinkworth, R. \& O'carroll, D. C. (2009) Robust models for optic flow coding in natural scenes inspired by insect biology. PLoS Computational Biology 5(11).

Brinkworth, R. S. A., Haltis, K. \& Sorell, M. (2010) A biologically inspired smart camera for use in surveillance applications. International Journal of Digital Crime and Forensics, Volume 2(3), p1-14.

Chen, Q.; Baldocchi, D.; Gong, P.; Kelly, M. Isolating individual trees in a savanna woodland using small footprint lidar data. Photogrammetry Engineering \& Remote Sensing 2006, Volume 72, p923-932.

Dowley, J. A., Dogancay, K. \& Brinkworth, R. (2013) A novel detection approach using bio-inspired vision for enhanced object tracking in video. In IEEE Eighth International Conference on Intelligent Sensors, Sensor Networks and Information Processing (ISSNIP) 2013, p497-502.

Furukawa, Y. \& Ponce, J. (2010) Accurate, dense, and robust multi-view stereopsis, IEEE Transactions in Pattern Analysis \& Machine Intelligence Volume 32, p1362-1376.

Griffiths, D. (2018) Biologically Inspired High Dynamic Range Imaging for use in Machine Vision, School of Engineering, University of South Australia, PhD.

Gong, P., Sheng, Y., Biging, G. (2002), 3D model-based tree measurement from high-resolution aerial imagery. Photogrammetry Engineering \& Remote Sensing, Volume 68, p1203-1212.

Hartley, R. (1997) In defense of the eight-point algorithm. IEEE Transactions of Pattern Analysis \& Machine Intelligence, Volume 19, p580-593.

Jones, H. G. (2004) Application of thermal imaging and infrared sensing in plantphysiology and ecophysiology. Advances in Botanical Research, Volume 41:107-163.

Kwak, D., Lee, W., Lee, J., Biging, G., Gong, P. (2007), Detection of individual trees and estimation of tree height using LiDAR data. Journal of Forest Research, 12, 425-434.
Longuet-Higgins, H. C. (1981) A computer algorithm for reconstructing a scene from two images. Nature.

Lowe, D. G. (1999) Object recognition from local scale-invariant features In Proceedings of IEEE International Conference on Computer Vision vol. 2, pp. 1150-1157.

Poursoltan, S. (2015) Bio-inspired vision model implementation in compressed surveillance videos. In Dept of Electrical Engineering.), Adelaide University, vol. $\mathrm{PhD}$.

Strigul, N., Pristinski, D., Purves, D., Dushoff, J., Pacala, S. (2008) Scaling from trees to forests: Tractable macroscopic equations for forest dynamics. Ecological Monographs, Volume 78, p523-545.

Strigul, N. Individual-Based Models and Scaling Methods for Ecological Forestry: Implications of Tree Phenotypic Plasticity, INTECH Open Access, Publisher Rijeka, Croatia, 2012.

Usamentiaga, R., Venegas, P., Guerediaga, J., Vega, L., Molleda, J. \& Bulnes, F. G. (2014) Infrared Thermography for Temperature Measurement and Non-Destructive Testing. Sensors 14(7):12305-12348.

Wallace, L.; Lucieer, A.; Watson, C.S. (2014), Evaluating tree detection and segmentation routines on very high resolution UAV LiDAR data, IEEE Transactions on Geoscience \& Remote Sensing, Volume 52, p7619-7628

Zarco-Tejada, P., Diaz-Varela, R., Angileri, V., Loudjani, P. (2014), Tree height quantification using very high resolution imagery acquired from an unmanned aerial vehicle (UAV) and automatic 3D photo-reconstruction methods, European Journal of Agronomy, Volume 55, p89-99

Revised February 2019 